\title{
A parts database with consensus parameter estimation for synthetic circuit design Supplemental information
}

\author{
Linh Huynh and Ilias Tagkopoulos* \\ Department of Computer Science 83 \\ UC Davis Genome Center \\ University of California \\ Davis, California 95616 \\ United States of America \\ E-mail: itagkopoulos@ucdavis.edu
}

\section{Running header}

A parts database with consensus parameter estimation for synthetic circuit design

Supplemental information 


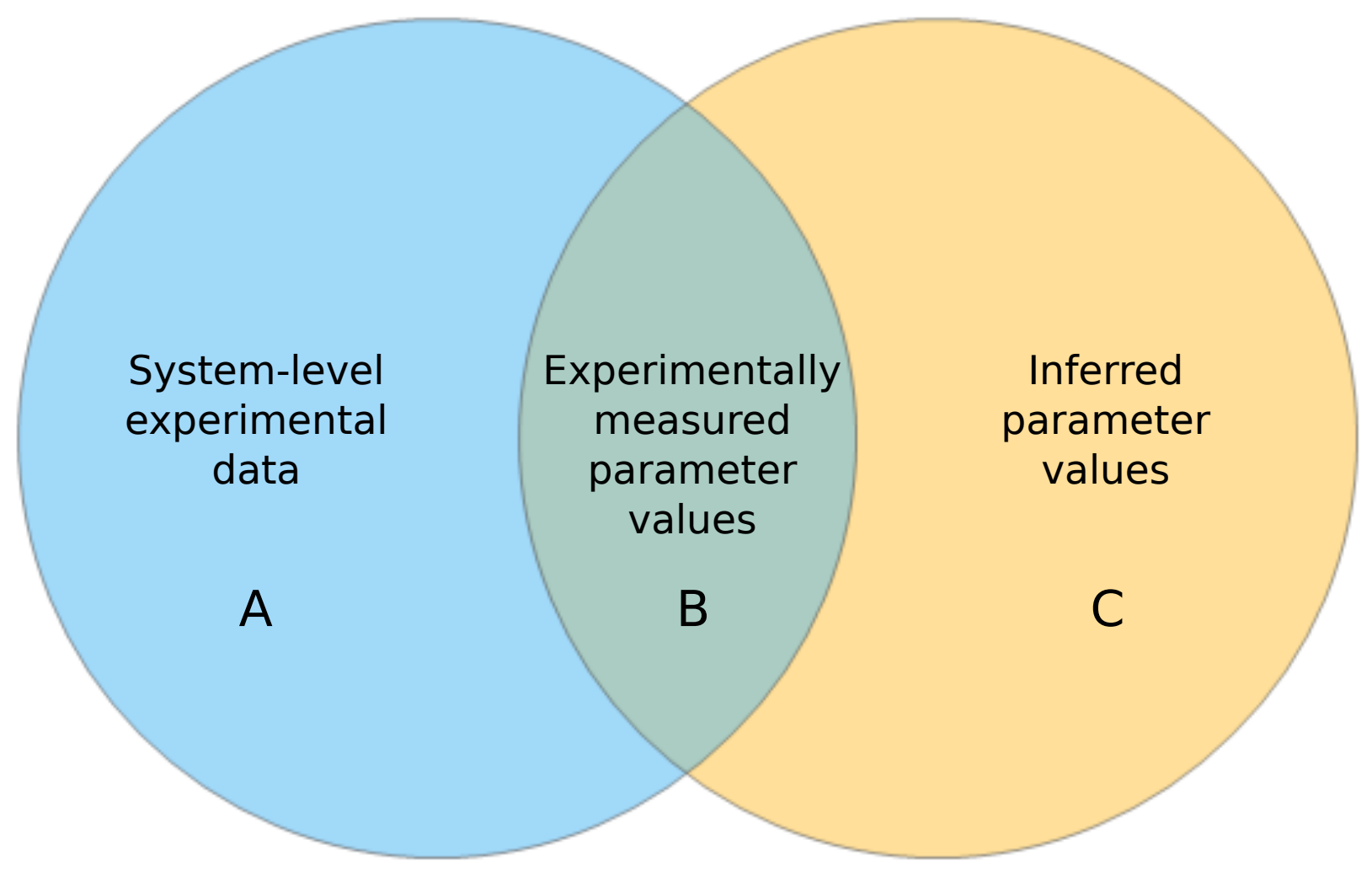

Figure S1: The overlap between experimental data (first circle) and the parameter values (second circle) are parameter values that are experimentally measured directly. By that way, we have three separate categories: (A) System-level experimental data experiments that are not designed to measure any parameter directly , (B) Experimentally measured parameter values, and (C) Inferred parameter values that are estimated from the systemlevel experimental data 


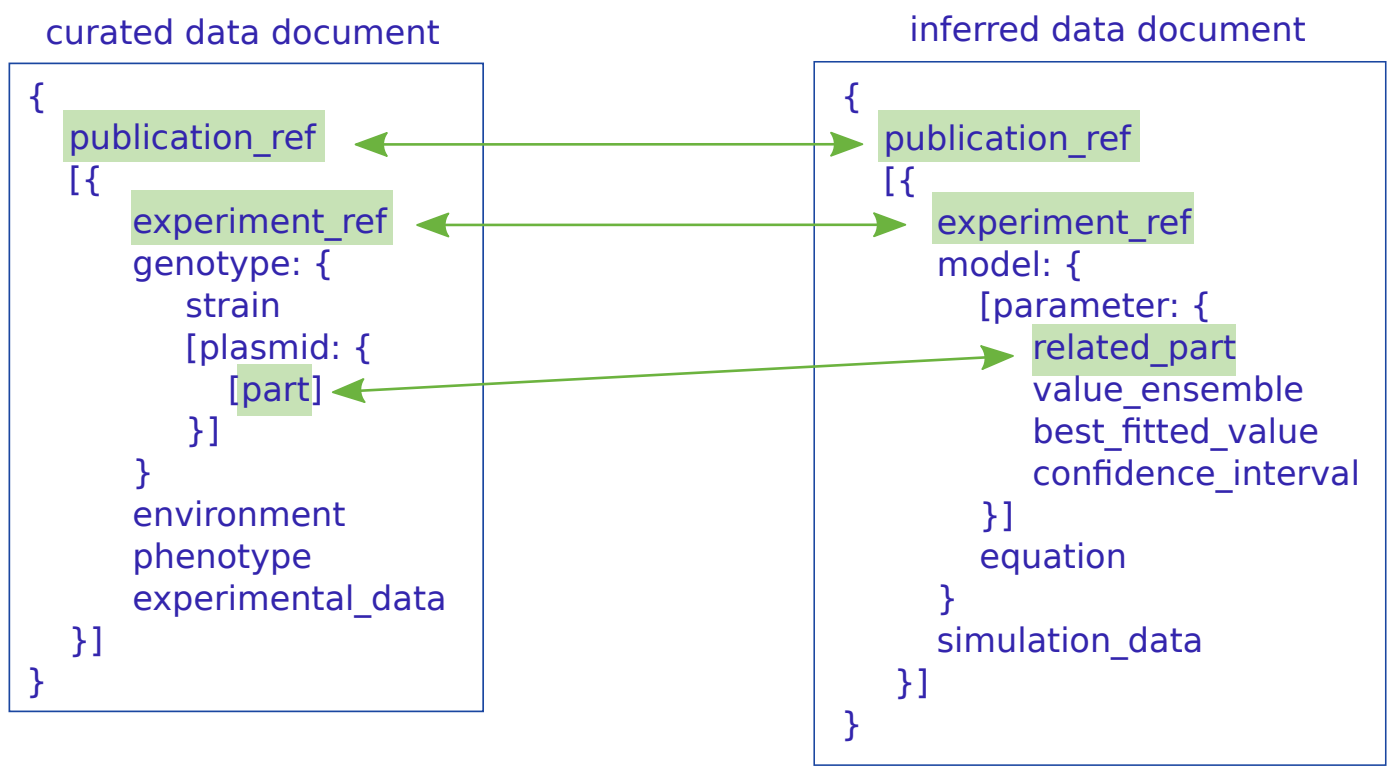

Figure S2: A physical design of PAMDB (document-oriented database). 


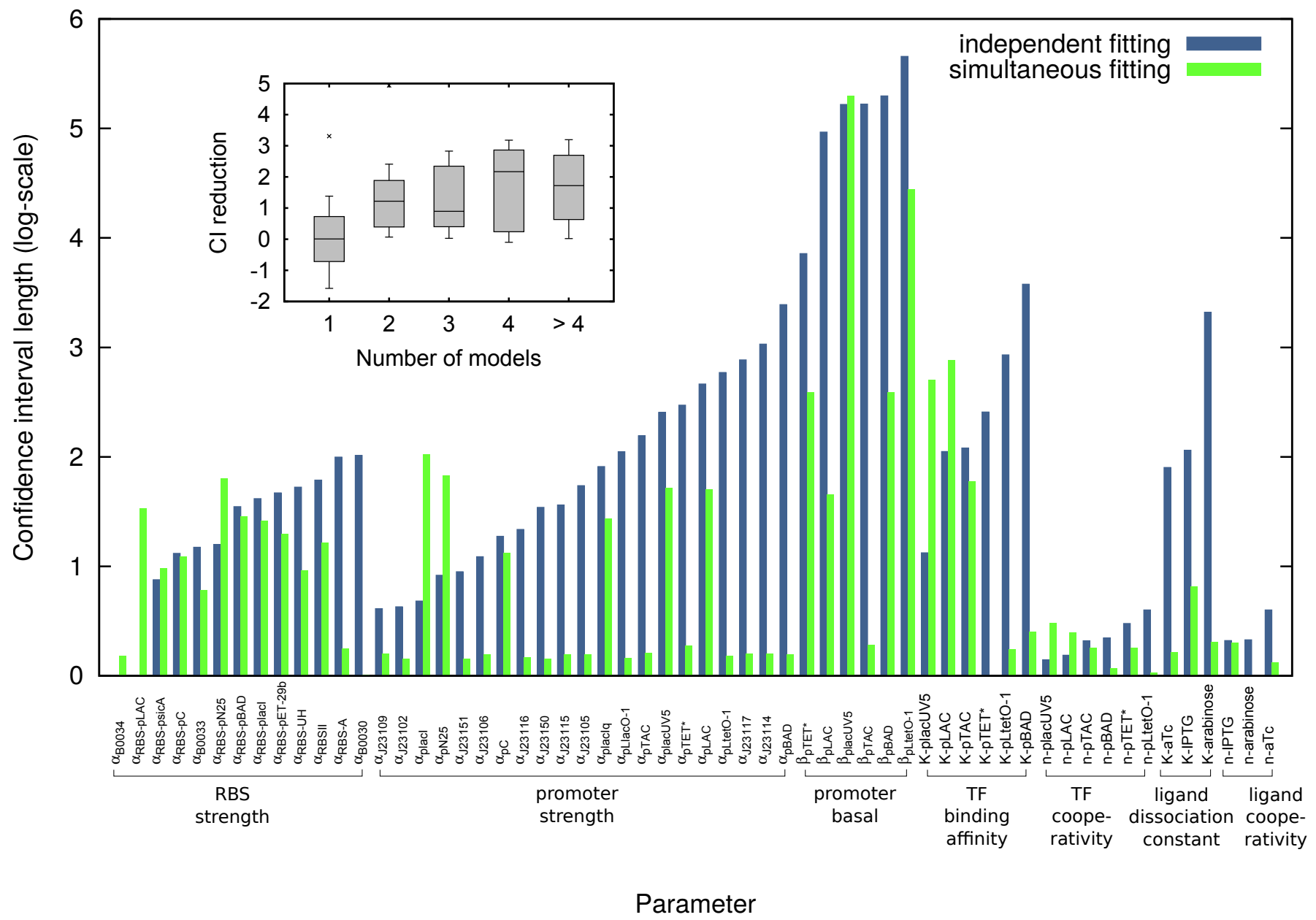

Figure S3: A comparison on the confidence interval length of parameter values between the independent fitting and the simultaneous fitting. 


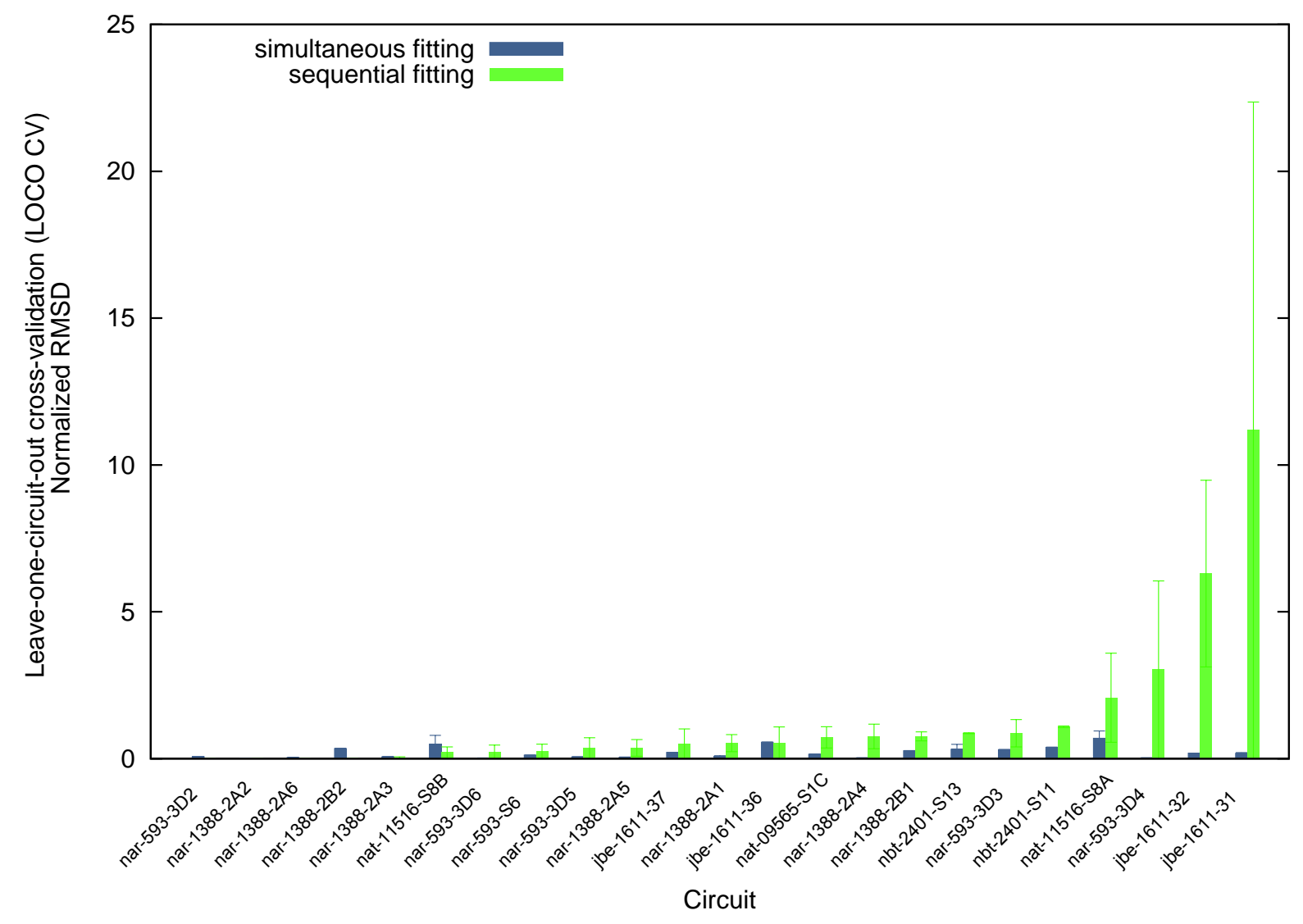

Figure S4: A comparison on the leave-one-circuit-out cross-validation (LOCO CV) normalized root-mean-square-deviation (Normalized RMSD) between the sequential fitting approach and the simultaneous fitting approach. 


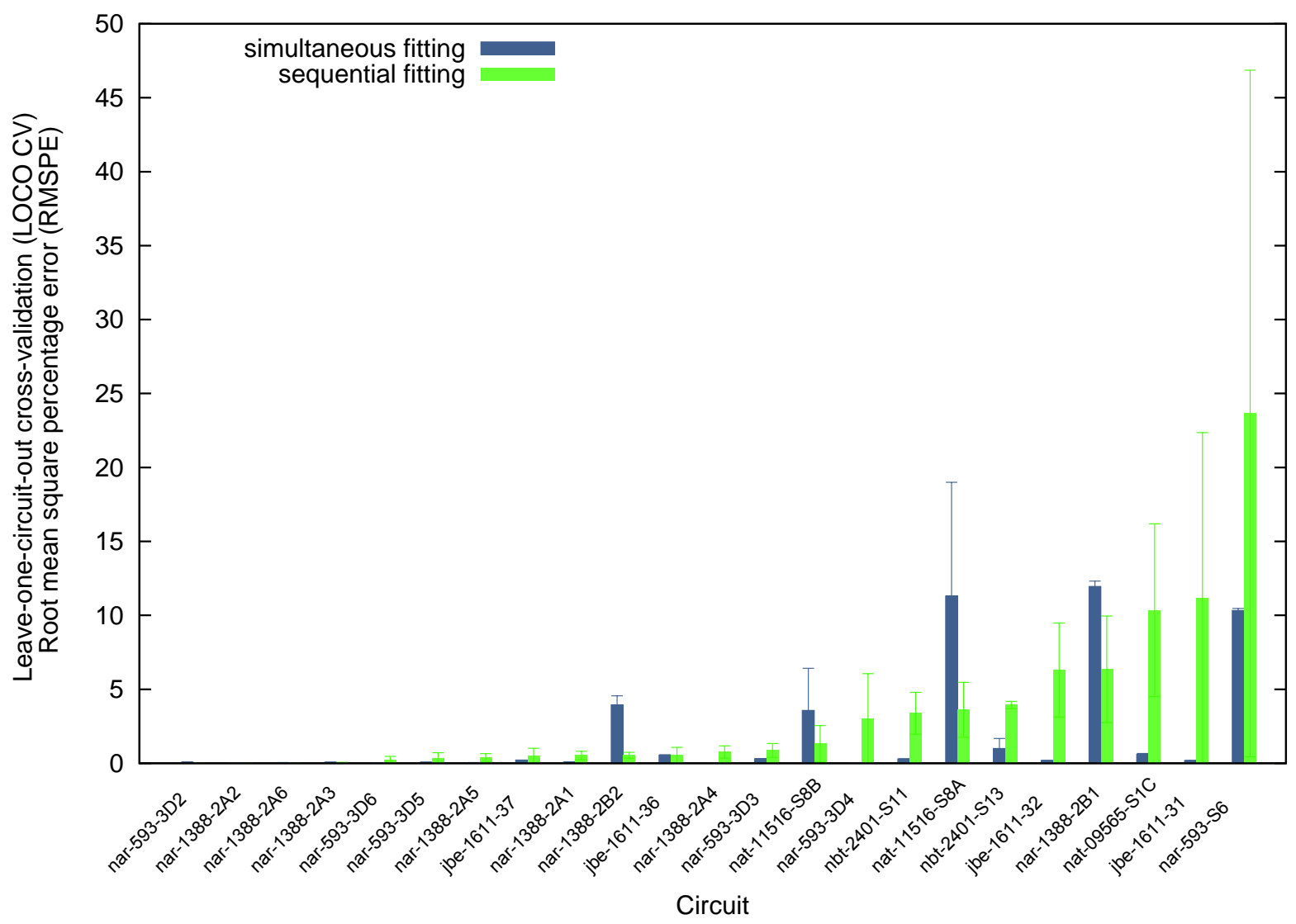

Figure S5: A comparison on the leave-one-circuit-out cross-validation (LOCO CV) root mean square percentage error (RMSPE) between the sequential fitting approach and the simultaneous fitting approach. 


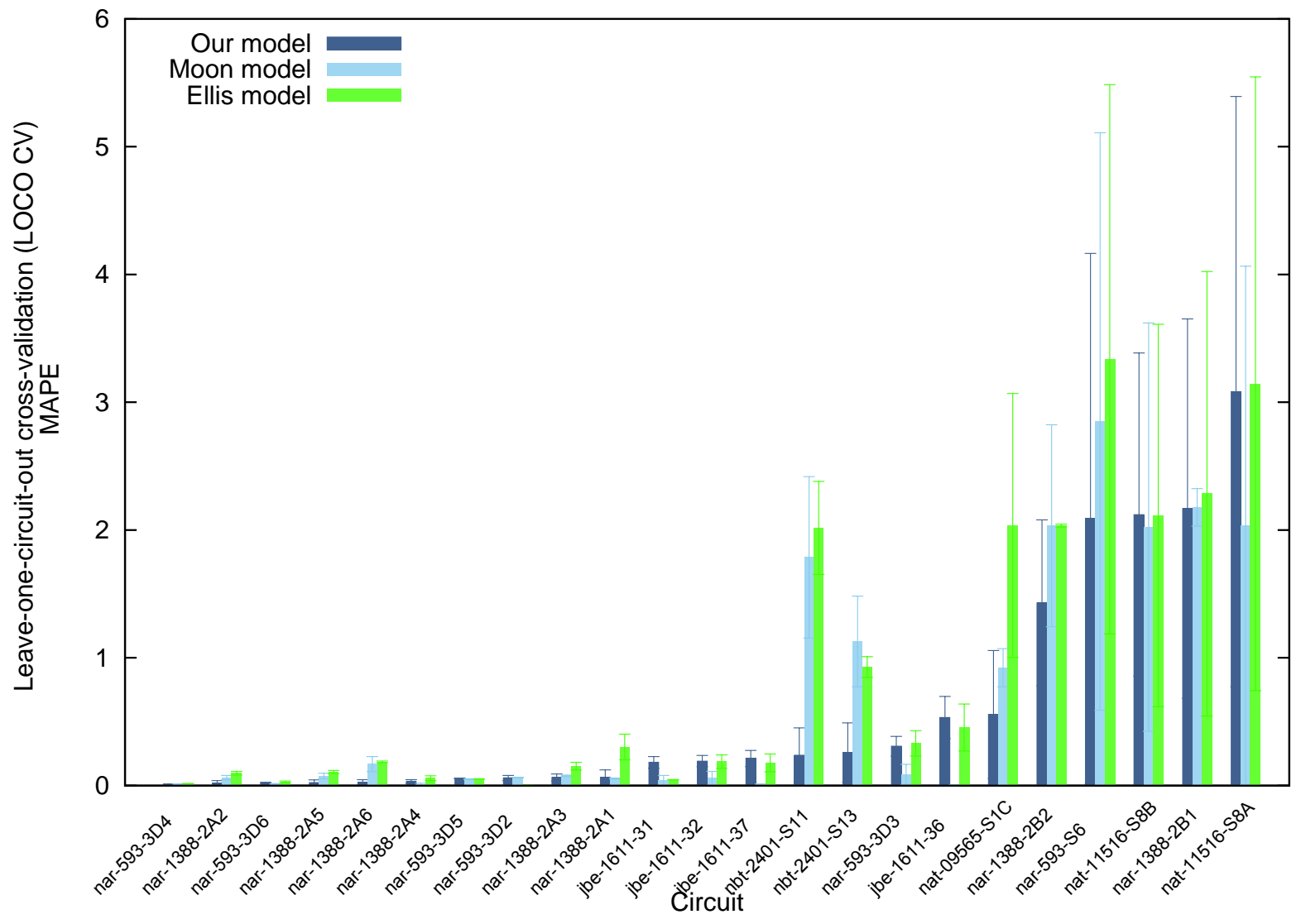

Figure S6: A comparison on the LOCO-CV MAPE of our model, Ellis et al. model (1) and Moon et al. model (2) 


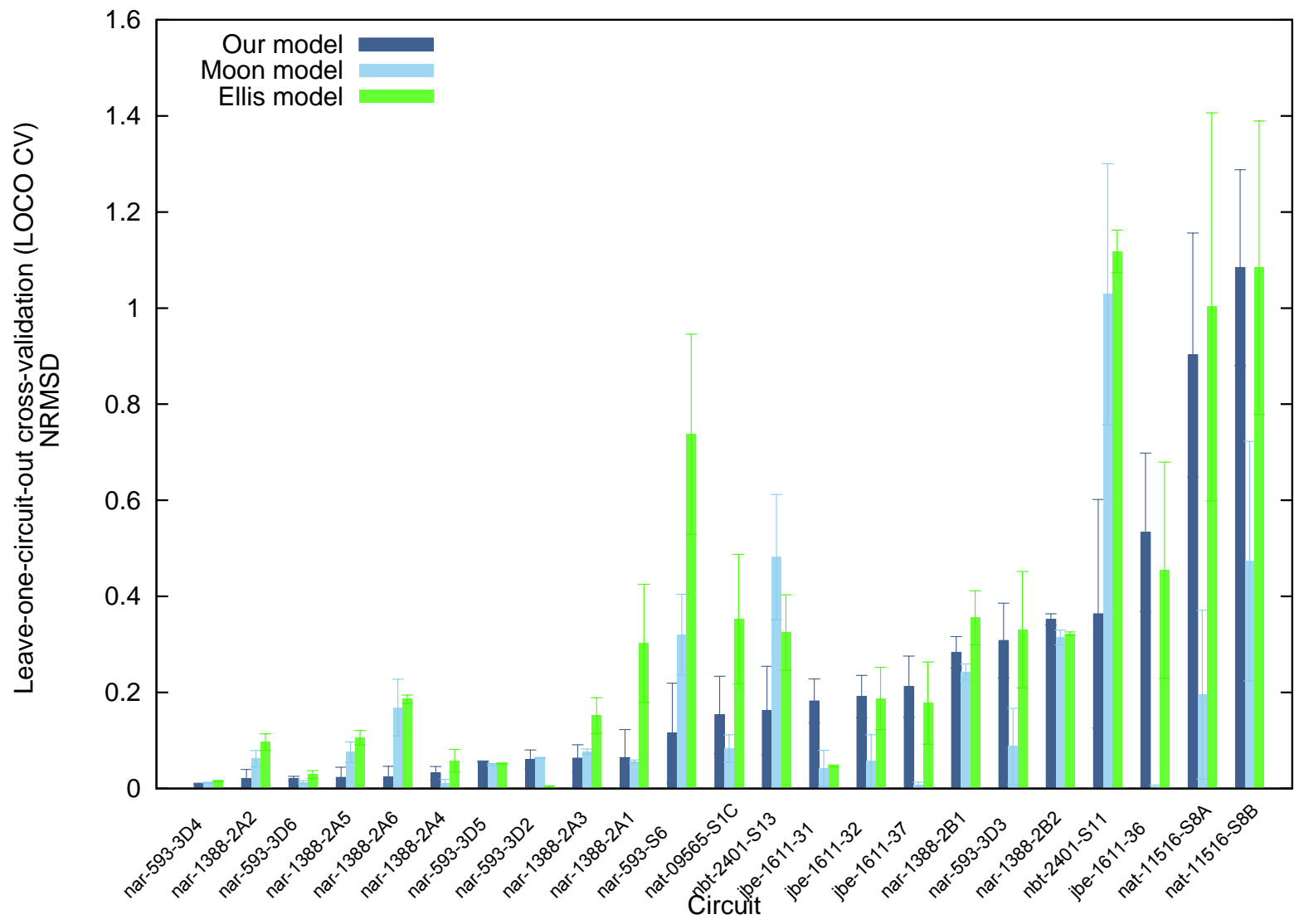

Figure S7: A comparison on the LOCO-CV Normalized RMSD of our model, Ellis et al. model (1) and Moon et al. model (2) 


\section{References}

1. Ellis, T., Wang, X., and Collins, J. J. (2009) Diversity-based, model-guided construction of synthetic gene networks with predicted functions. Nature biotechnology 27, 465-471.

2. Moon, T. S., Lou, C., Tamsir, A., Stanton, B. C., and Voigt, C. A. (2012) Genetic programs constructed from layered logic gates in single cells. $\mathrm{Na}$ ture 491, 249-253. 